Research Article

\title{
Tactile Sensor Array with Fiber Bragg Gratings in Quasi-Distributed Sensing
}

\author{
Marcelo A. Pedroso $\left(\mathbb{D},{ }^{1}\right.$ Lucas H. Negri $\left(\mathbb{D},{ }^{2}\right.$ Marcos A. Kamizi $\mathbb{D}^{1},{ }^{1}$ José L. Fabris $\mathbb{D},^{1}$ \\ and Marcia Muller $\left(\mathbb{D}^{1}\right.$ \\ ${ }^{1}$ Graduate Program in Electrical and Computer Engineering, Federal University of Technology-Paraná, Curitiba, PR, Brazil \\ ${ }^{2}$ Federal Institute of Education, Science and Technology of Mato Grosso do Sul, Aquidauana, MS, Brazil \\ Correspondence should be addressed to José L. Fabris; fabris@utfpr.edu.br
}

Received 30 August 2017; Accepted 14 December 2017; Published 13 February 2018

Academic Editor: Carlos Ruiz

Copyright (c) 2018 Marcelo A. Pedroso et al. This is an open access article distributed under the Creative Commons Attribution License, which permits unrestricted use, distribution, and reproduction in any medium, provided the original work is properly cited.

\begin{abstract}
This work describes the development of a quasi-distributed real-time tactile sensing system with a reduced number of fiber Bragg grating-based sensors and reports its use with a reconstruction method based on differential evolution. The sensing system is comprised of six fiber Bragg gratings encapsulated in silicone elastomer to form a tactile sensor array with total dimensions of $60 \times 80 \mathrm{~mm}$, divided into eight sensing cells with dimensions of $20 \times 30 \mathrm{~mm}$. Forces applied at the central position of the sensor array resulted in linear response curves for the gratings, highlighting their coupled responses and allowing the application of compressive sensing. The reduced number of sensors regarding the number of sensing cells results in an undetermined inverse problem, solved with a compressive sensing algorithm with the aid of differential evolution method. The system is capable of identifying and quantifying up to four different loads at four different cells with relative errors lower than $10.5 \%$ and signal-tonoise ratio better than $12 \mathrm{~dB}$.
\end{abstract}

\section{Introduction}

Some works have pointed to the use of arrays of fiber Bragg grating- (FBG-) based transducers in tactile sensing systems (TSS) applied to the mapping of forces in robotic systems [1], biomedical [2], and medical areas [3, 4]. These systems make use of the FBG multiplexing capability. Despite the limitations in the number of FBG that can be multiplexed, FBG-based sensors enable multipoint monitoring in a quasi-distributed configuration.

Different approaches have been proposed for the development of sensor arrays. Such strategies include gluing grating-based transducers directly under the surface of a steel or polymethyl methacrylate (PMMA) plate $[5,6]$, attaching gratings to iron rings [7], encapsulating FBGs in blocks of silicone positioned under a steel plate [8], or forming an array of sensors in a single silicone encapsulation $[1,9]$.

The embedding of many FBG in a single thin sheet of a host material makes the tactile sensor array (TSA) flexible, so that it can be adapted to surfaces with different forms. Among the manufacturing methods, molding is a low-cost process that allows an easy encapsulation of a set of FBG sensors [9].

Some tactile sensing systems use one sensor element dedicated to each point of sensing, requiring an increased number of elements if the area of monitoring is wide. Under these conditions, the cost and complexity of the sensing system also increase. Nevertheless, this number can be reduced if the responses of the sensor elements in the TSS are coupled, making such a system less expensive and more robust [6].

Computational methods as artificial neural networks $[10,5]$ and fuzzy rule-based systems [6] have been used to interpret data from sensing systems. In an effort to further reduce the number of elements of a sensing array, Negri et al. [7] reported the development of a tactile sensing system with an array of sensors in a quasi-distributed configuration. In this system, a metal plate was divided into nine square regions monitored by seven FBGs installed in metal rings. The application of compressive sensing theory [11] to the underdetermined inverse problem resulting from this 
configuration showed the reliability of the method application in systems with a sparse solution. The least absolute shrinkage and selection operation (LASSO) [12] algorithm based on compressive sensing (CS) used to solve the problem provided a reconstruction ratio (RR) of $73.3 \%$ for three loads simultaneously applied on the metal surface.

In 2017, Negri et al. [8] developed a sensing system composed of a steel plate instrumented with eight sensor elements glued under its surface. Sixteen sensing cells were preestablished on the surface of the plate. The coupled responses of the sensors, each one corresponding to one FBG embedded in a small silicone block, allowed the application of CS. A sparse differential evolution (SDE) method was implemented to solve the inverse problem, leading to a RR of $84 \%$ with the application of three simultaneous loads.

Devices based on different principles of operation as resistive strain gauges [13], piezoresistive [14], and capacitive sensors [15] have been used in tactile sensor systems (TSS). Despite the benefits of these traditional technologies, they are not suitable for applications that require the interconnection of many sensor elements, low power consumption, and high immunity to electromagnetic interference [4]. In this sense, the outstanding characteristics of FBG sensors such as reduced size and weight, flexibility, easiness of encapsulation and multiplexing, low loss transmission, high immunity to electromagnetic interference, resistance to temperature, and electrical passivity [16] have been successfully explored.

In this work, the SDE method was used to reconstruct up to four loads applied on the surface of a TSA composed of six FBGs embedded in a single thin and flat sheet of silicone elastomer. The paper is an extended version of a previous work that describe the details of the sensor array manufacture [9]. Silicone has adequate properties that allows its use in TSS, such as flexibility, improved tactile response, fast cure process at room temperature, and, in addition, low fabrication cost. To shape the array, it was used as a mold manufactured with 3D printing technology, which provides the FBGs positioning in the sensing device with high accuracy $( \pm 0.1 \mathrm{~mm})$ [9]. Despite the use of the FBG-coupled responses, a necessary condition for the application of CS, the sensing array works based on the detection of loads applied to specific predetermined regions of the TSA surface. Therefore, the TSA is still considered a quasi-distributed sensing system. The fast response time of the whole system allows its use in real-time applications.

\section{Materials and Methods}

2.1. FBG-Based Transducers. Fiber Bragg gratings were used in this work owing to their unique advantageous characteristics and the capacity to detect mechanical deformations [16].

Longitudinal deformations and temperature variations produce changes in the effective refractive index $\left(n_{\text {eff }}\right)$ of the FBG, as well as in the spatial period between the grating planes $(\Lambda)$, resulting in shifts of the Bragg wavelength $\left(\lambda_{\mathrm{B}}\right)$ given by the following:

$$
\lambda_{\mathrm{B}}=2 n_{\mathrm{eff}} \Lambda
$$

As the FBG shows cross-sensitivity to deformation and temperature, it is necessary to compensate such effect in order to measure only mechanical deformations. If the temperature is kept constant, the response of the FBG to longitudinal deformation is given by the following:

$$
\Delta \lambda_{\mathrm{B}}=\lambda_{\mathrm{B}}\left\{1-\frac{n^{2}}{2}\left[p_{12}-v\left(p_{11}+p_{12}\right)\right] \varepsilon_{\mathrm{z}}\right\},
$$

where $p_{11}$ and $p_{12}$ are the elastooptic coefficients, $\varepsilon_{\mathrm{z}}$ is the relative longitudinal deformation (strain) applied to the FBG, and $v$ is the Poisson coefficient of silica [16].

In this work, all FBGs were written in standard single mode fiber (SSMF, DRAKTEL, G-652) by the exposition to laser light diffracted by a phase mask [17]. The writing system at the Photorefractive Devices Unit of the Federal University of Technology-PR (UTFPR) uses an excimer Argon Fluoride laser (Coherent Xantos, $193 \mathrm{~nm}, 2.5 \mathrm{~mJ}$ pulse energy, $250 \mathrm{~Hz}$, exposure time of $\sim 1$ minute). Six different phase masks from Ibsen Photonics were used to fabricate six FBGs with resonances at 1520.807, 1523.550, 1526.687, $1530.637,1534.128$, and $1537.619 \mathrm{~nm}$ at $22.0 \pm 0.5^{\circ} \mathrm{C}$. All FBGs have reflectivity lower than $15 \%$, length of approximately $3 \mathrm{~mm}$, spectral bandwidth of $0.21 \mathrm{~nm}$, and sensitivity of $9.8 \mathrm{pm} /{ }^{\circ} \mathrm{C}$ and $1.13 \mathrm{pm} / \mu \varepsilon$.

2.2. Tactile Sensor Array Production. The first stage of the TSA production starts with the adequate choice of the host material used to embed the FBG. A room temperature vulcanizing (RTV) silicone (Down Corning, BX3-8001) was chosen as embedding material considering its suitable properties [18] and the facility of using the molding method. Additionally, the FBG must be protected against extreme deformations as the array of sensors is submitted to mechanical stress. Therefore, a 1:1 proportion of silicone and quartz powder was used to increase the hardness of the TSA. This proportion proved to be adequate to keep the integrity of the FBG without impairing the flexibility of the sensor array for the preestablished dynamic range of loads [19].

To shape the tactile sensor array and also to dispose the six FBGs in the middle of the silicone sheet thickness, it used a 3D-printed mold produced from a model created by a computer-aided-design (CAD) software. This fabrication method allows the production of narrow slits at the mold walls, which are used for positioning the segment of the optical fiber containing the FBG in the desired place. In addition, with the use of acrylonitrile butadiene styrene (ABS) filament $(1.75 \mathrm{~mm})$ to print the mold, there is nonadhesion with the RTV silicone, resulting in an easy process of demolding.

The mold is a rectangular box with internal dimensions of $75 \times 105 \mathrm{~mm}$ and walls $5 \mathrm{~mm}$ high. The slits in the opposite walls are $2.5 \mathrm{~mm}$ deep and $15 \mathrm{~mm}$ apart from each other, as shown in Figure 1.

The six optical fiber segments, each one containing a single FBG, are positioned at the mold using the slits. FBGs are distributed in order to keep the gratings approximately $25 \mathrm{~mm}$ apart. After this process, the mold was fixed on a flat and stiff surface. Then, the fiber segments were stretched one by one and the loose tips were fixed on the flat surface with 


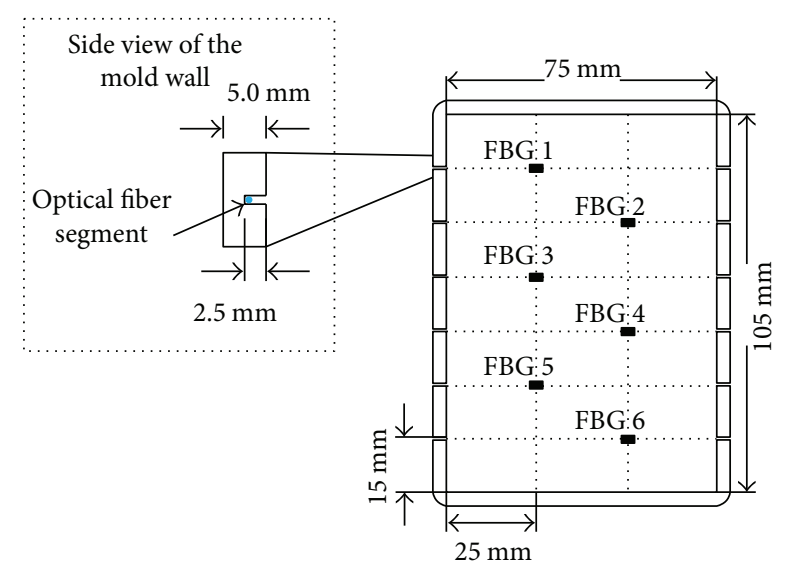

FIGURE 1: Schematic representation of the FBGs at the 3D-printed mold and a detail of the slits in the mold walls.

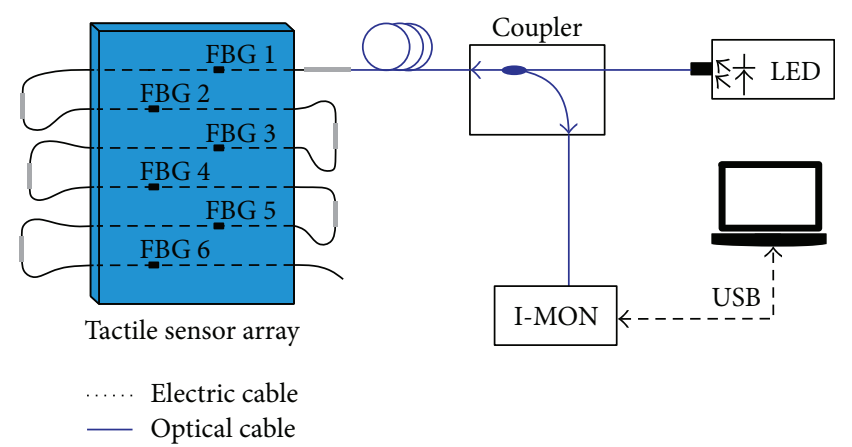

FIgURE 2: Tactile sensing system (TSS) and the interrogation unit.

adhesive tape. Finally, the silicone mixture with quartz was carefully spread out in the mold forming a homogeneous layer embedded with the FBGs.

The vulcanization process occurs at room temperature along 24 hours; after this period, the TSA is easily removed from the mold. The TSA surface kept in contact with the mold is free from irregularities and is used as the sensing surface. Afterwards, the tips of the optical fiber segments were connected generating a set of six in-series FBGs (Figure 2).

2.3. Interrogation System. For the TSA interrogation, one of the free fiber tips is connected to a superluminescent LED (Superlum PILOT-2, centered at $1558.2 \mathrm{~nm}$, spectral bandwidth of $73.8 \mathrm{~nm}$ ) via an optical coupler. The reflected spectra of the FBG sensors are measured by an optical interrogation monitor (Ibsen, I-MON 512E, $970 \mathrm{~Hz}$ maximum sampling rate) with resolution smaller than $0.5 \mathrm{pm}$. All spectral data are sent to a computer that records the Bragg wavelengths and their relative shifts. Figure 3 shows the spectrum of the six FBGs in the TSA.

2.4. Characterization Procedure. For the TSA assessment by means of the FBGs' repeatability and linearity, loads up to $250 \mathrm{~g}$ were applied at the central position of the array, in steps of $25 \mathrm{~g}$ with delay of approximately $5 \mathrm{~s}$, using the $Z$-stage load system shown in Figure 4. The FBG responses were measured

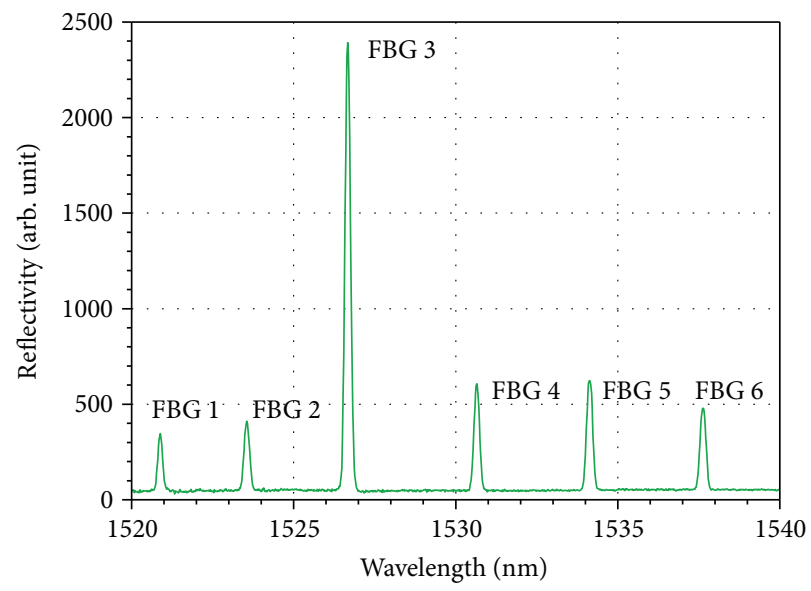

FIgURe 3: Spectrum of the six FBGs in the TSA recorded with an optical spectrum analyzer (Anritsu, OSA MS9710b, 1001 samples, $0.1 \mathrm{~nm}$ resolution).

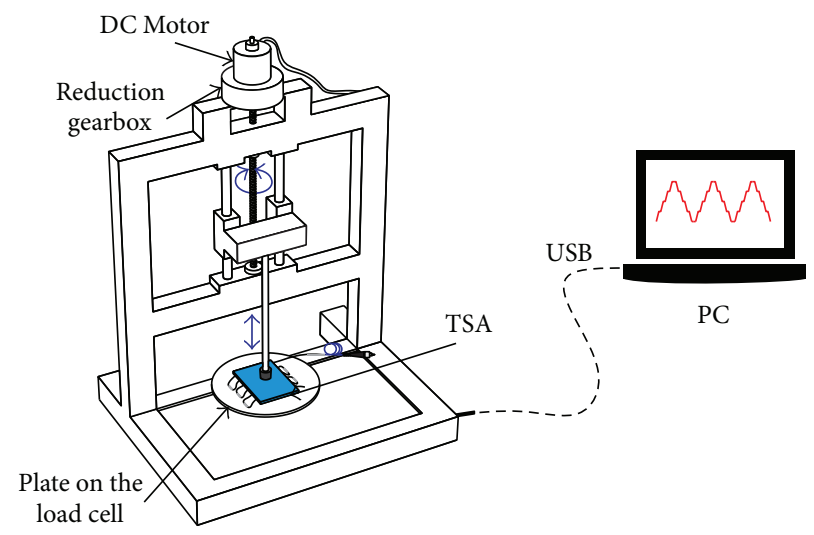

FIgURE 4: Schematic representation of the $Z$-stage load system.

under repeatability conditions with 15 acquisitions and under intermediate precision conditions along three upand-down cycles [20].

The same previously described methodology was applied to a single FBG of the TSA, and the complete metrological characteristics were determined, including hysteresis, sensitivity, resolution, and linearity $[19,20]$. The sensor resolution is the ratio between the resolution of the optical interrogation monitor (in $\mathrm{pm}$ ) and the sensor sensitivity (in $\mathrm{pm} / \mathrm{g}$ ). The linearity is the maximum absolute deviation of the experimental data points regarding the calibration curve. Finally, the hysteresis is the maximum value obtained by the summation of absolute deviations of the experimental points regarding the calibration curve.

The TSA linearity was evaluated in three tests in which loads were applied both individually and simultaneously on the sensor array to verify the additivity property. It is expected for a linear system that the summation of the individual responses matches the response obtained with the simultaneous application of those loads [7]. Temperature was kept at $22.0 \pm 0.5^{\circ} \mathrm{C}$ during the characterization experiments. 


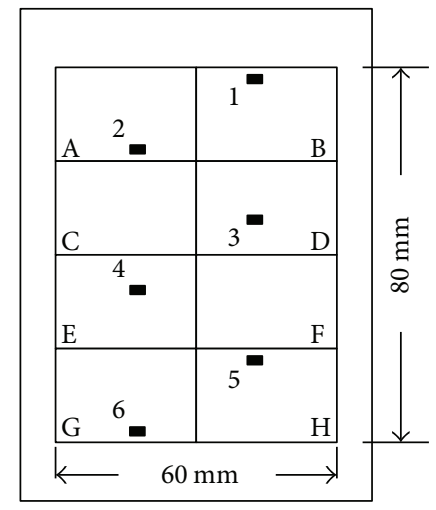

Figure 5: Schematic representation of the TSA divided into eight sensing cells.

2.5. Signal Reconstruction. The TSA was divided into eight rectangular regions (sensing cells labeled from $\mathrm{A}$ to $\mathrm{H}$ ) with dimensions of $20 \times 30 \mathrm{~mm}$, as shown in Figure 5 .

As sensitivity depends on the distance between the load application and the sensor element, the size of the sensing area was defined in order to guarantee the coupling between the six FBG responses [9]. The six FBGs (labeled from 1 to 6 in Figure 5) monitor 8 sensing cells, resulting in an undetermined inverse problem. This problem can be solved by considering the ideal case of a linear response from the FBGs using the following:

$$
\mathbf{A x}=\mathbf{y},
$$

where $\mathbf{x}$ is a vector with $n=8$ elements containing the values of the loads applied to each sensing cell, $\mathbf{y}$ is a vector with $m=6$ elements that correspond to the Bragg wavelength shifts, and $\mathbf{A}$ is the $m \times n$ sensing matrix that express the relation between load positions and signals from the sensors. If $m<n$, the linear system is undetermined and can have an infinite number of solutions. However, if the vector $\mathbf{x}$ is sparse, optimization methods, such as CS with the aid of DE can be used to solve this problem [8].

The optimization method used to solve the problem in (3) is given by the following:

$$
\begin{aligned}
& \min _{\mathbf{x}}\|\mathbf{A x}=\mathbf{y}\|_{2}^{2}+\sigma\|\mathbf{x}\|_{p}^{p} \\
& \text { subject to } 0 \leq \mathbf{x} \leq \text { ulimit, }
\end{aligned}
$$

where the parameter $\sigma$ is a trade-off between the fidelity of reconstruction $\left(\|\mathbf{A} \mathbf{x}=\mathbf{y}\|_{2}^{2}\right)$ and the sparsity of the solution $\left(\|\mathbf{x}\|_{p}^{p}\right) ; p$ was used for the term associated with the sparsity of the solution, and ulimit $=0.250(250 \mathrm{~g})$ is the maximum value of the applied loads. The value of $p=0.01$ was used to perform a smooth approximation to the $l_{0}$-norm. The solution of (4) is given by the DE algorithm in scheme $D E$ / rand/1/bin with 150 individuals and a maximum number of 1500 interations.

2.6. Reconstruction Tests. First, a set of validation tests, containing 22 values measured with the application of up to three loads (200 g, $100 \mathrm{~g}$, and $50 \mathrm{~g}$ ) on the TSA, was used to determine the parameters $\sigma$ [8]. Then, reconstruction tests were carried out for different combinations of loads. A set of 8 measurements were carried out with each one of the 3 loads resulting in a data set containing 24 values. A total of 20 different configurations of cells were randomly chosen for the simultaneous application of 2,3, and 4 loads resulting in 60 new values, totalizing 84 reconstruction tests.

The metallic cylindrical loads with different masses have also different cross-sections. Therefore, circular-shaped elements with $20 \mathrm{~mm}$ of diameter were positioned on the TSA surface in order to create a unique contact area.

After all tests, the signal-to-noise ratio (SNR) was used as a metric to evaluate the matching between the reconstructed signals and the actual applied loads $[7,8]$. In this work, the SNR is defined by the following:

$$
\mathrm{SNR}=20 \log _{10}\left(\frac{\left\|\mathbf{x}_{0}\right\|}{\left\|\mathbf{x}-\mathbf{x}_{0}\right\|}\right)
$$

where $\mathbf{x}_{0}$ is the vector of the actual loads and $\mathbf{x}$ is the resulting reconstruction. Better performances of the sensor array are associated with higher ratios between the reconstruction and the original signal. To assess the percentage of the cases that were successfully recovered, a threshold of $12 \mathrm{~dB}$ was considered as a minimal requirement and the reconstruction rate (RR) represents this percentage.

The sparse differential evolution (SDE) depends on the knowledge of the sensing matrix $\mathbf{A}$, which was experimentally obtained by the sequential and individual application of a $200 \mathrm{~g}$ load at the $n=8$ cells of the TSA. Each cell corresponds to a column in the sensing matrix. The $m=6$ elements of a column are filled with the sensitivities of the six FBGs when the load is applied to one cell. The sensors sensitivities were computed by the ratio between the applied load and the corresponding Bragg wavelength shift. As a final step, sensitivities are normalized so that the search for the sparsest solution does not benefit sensors with higher sensitivities, which could cause the system convergence to a wrong result [8].

\section{Results and Discussion}

The application of loads at the central position of the TSA resulted in wavelength shifts of all FBGs. This behavior indicates a coupled response among the FBGs, allowing its application in a quasi-distributed sensing system. An important feature is the linearity of the FBG responses that allows the use of the optimization method described in (4).

As shown in Table 1, sensitivities depend on the distance between the load application and the position of the FBGs. All FBG sensitivities and the standard error of the mean (SEM) obtained by the application of loads at the central position of the TSA are shown in Table 1.

FBGs 3 and 4, the FBGs closest to the position of the load application, showed the highest sensitivities, as can be seen in Table 1. On the other hand, FBGs 1, 2, 5, and 6 are less sensitive, as expected.

As the positioning of the segments of fiber containing the FBGs in the mold is manually controlled, small differences in 
TABLE 1: FBG sensitivities for loads applied at the central position of the TSA.

\begin{tabular}{lccc}
\hline Sensor & Sensitivity $(\mathrm{pm} / \mathrm{g})$ & $\begin{array}{c}\text { Distance } \\
\text { of the load } \\
\text { application }(\mathrm{mm})\end{array}$ & $R$-coefficient \\
\hline 1 & $0.0327 \pm 0.0004$ & 39.53 & 0.999 \\
2 & $0.0661 \pm 0.0004$ & 25.74 & 0.999 \\
3 & $\mathbf{0 . 1 9 5 8} \pm \mathbf{0 . 0 0 2 7}$ & 14.58 & 0.999 \\
4 & $\mathbf{0 . 1 8 0 1} \pm \mathbf{0 . 0 0 4 1}$ & 14.58 & 0.997 \\
5 & $0.0455 \pm 0.0007$ & 25.74 & 0.998 \\
6 & $0.0183 \pm 0.0003$ & 39.53 & 0.998 \\
\hline
\end{tabular}

TABLE 2: Metrological characteristics of one FBG in the TSA.

\begin{tabular}{lc}
\hline Metrological characteristics & Encapsulated FBG \\
\hline Measuring interval $(\mathrm{g})$ & $0-250$ \\
$R$-coefficient & 0.99 \\
Sensitivity (pm/g) & $0.371 \pm 0.003$ \\
Resolution (g) & $1.35 \pm 0.39$ \\
Linearity (\%) & \pm 2.42 \\
Hysteresis (\%) & \pm 4.33 \\
\hline
\end{tabular}

the distance of the gratings with respect to the point of load application result in different sensitivities for these gratings. Furthermore, the 3D printer has $0.10 \mathrm{~mm}$ of resolution, which results in slits with $0.25 \pm 0.10 \mathrm{~mm}$.

It is well known that a nonuniform distribution of mechanical deformation along the length of an FBG leads to a chirping, which deforms the spectrum of the fiber Bragg grating [1]. In this work, applied loads were up to $250 \mathrm{~g}$ leading to a nonchirped spectrum for all FBGs. Moreover, the spectral separation between the FBG resonances $(\sim 3 \mathrm{~nm})$ avoids spectral overlapping.

An overall understanding of the behavior presented by the FBGs embedded in the silicone elastomer is necessary to optimize the sensor. Therefore, for a complete characterization, the previous test was replicated; however, the loads were applied directly at the position of one of the FBGs in the TSA. The complete metrological characteristics are shown in Table 2.

Linearity and hysteresis were calculated with respect to the difference between the upper and lower limits (span) of the measuring interval [19]. The hysteresis contribution is the principal cause of the dispersion in the measurements.

Searching for a complete assessment of the TSA linearity, tests of additivity were carried out and the results are shown in Figures 6, 7, and 8. The square symbols represent the simultaneous application of the loads, and the stacked bars represent the individual application.

Tests of additivity resulted in errors lower than $31.5 \%$ for FBGs close to the applied loads. For the FBGs far away from the point of the load application, errors of up to $92 \%$ were obtained. Hysteresis and thermal expansion were the main sources of error. Figure 6 shows that the majority of the FGBs are sensitive even to loads applied on cells B and G located on the TSA edges. As expected, loads applied close to each other

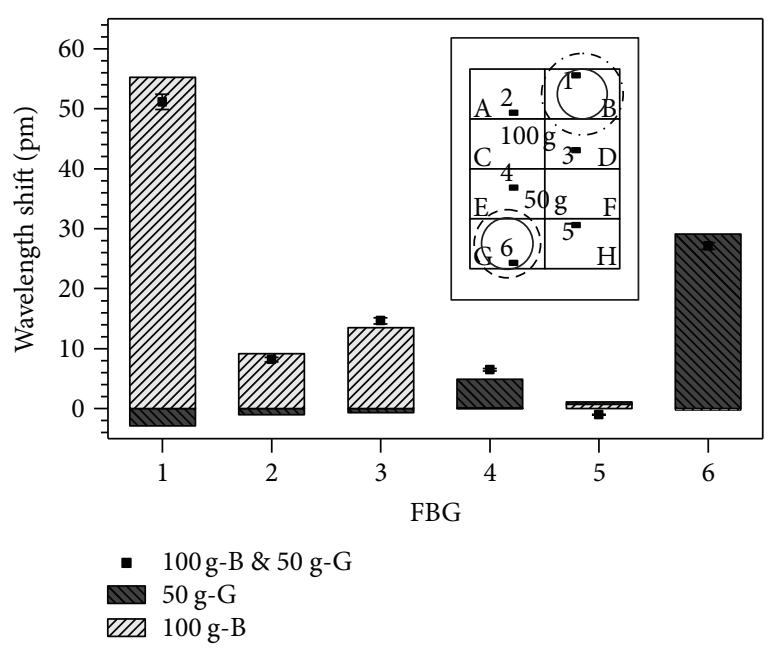

FIgURE 6: Sensor responses to loads applied individually (stack columns) and simultaneously (square symbols) on cells B and G. The inset indicates the position of the loads in a diagram of the TSA.

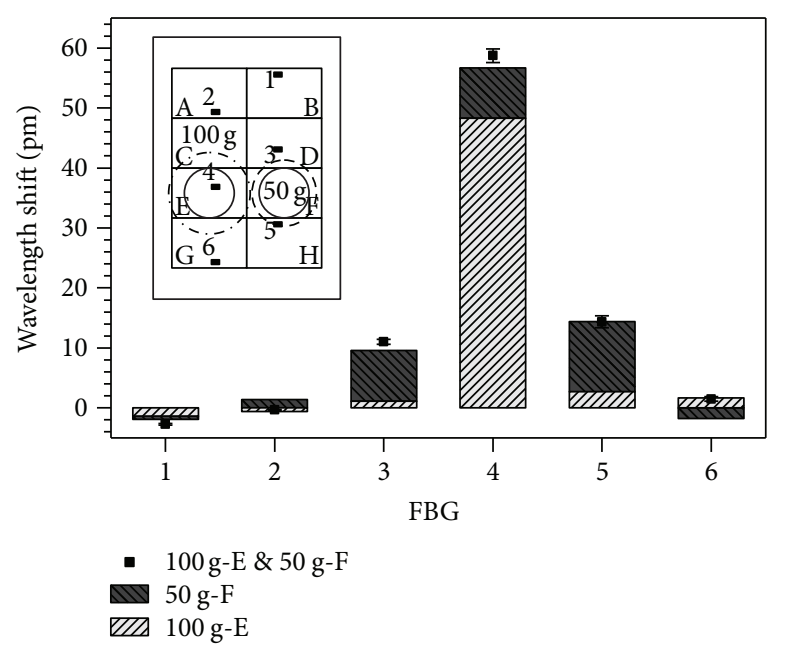

FIgURE 7: Sensor responses to loads applied individually (stack columns) and simultaneously (square symbols) on cells E and F. The inset indicates the position of the loads in a diagram of the TSA.

(see Figure 7) produce significant coupled responses for the FBGs close to the load positions. Finally, Figure 8 shows the FBG responses for three loads simultaneously applied on the TSA.

The TSA performance was assessed with the reconstruction tests carried out with 1,2,3, and 4 loads. As previously stated, the TSA was divided into eight sensing cells, each one with $30 \times 20 \mathrm{~mm}$, and different configurations of load application were tested.

The ulimit for the SDE method was $0.250(250 \mathrm{~g})$; in other words, the reconstruction algorithm only recognizes applied loads from 0 to $250 \mathrm{~g}$. This approach is used to limit the search space and optimize the results. Negri et al. have shown that an adequate ulimit can improve the quality of the reconstruction [8].

Figure 9 shows the best and worst reconstructions among the tests realized with three loads. The smallest SNR of 


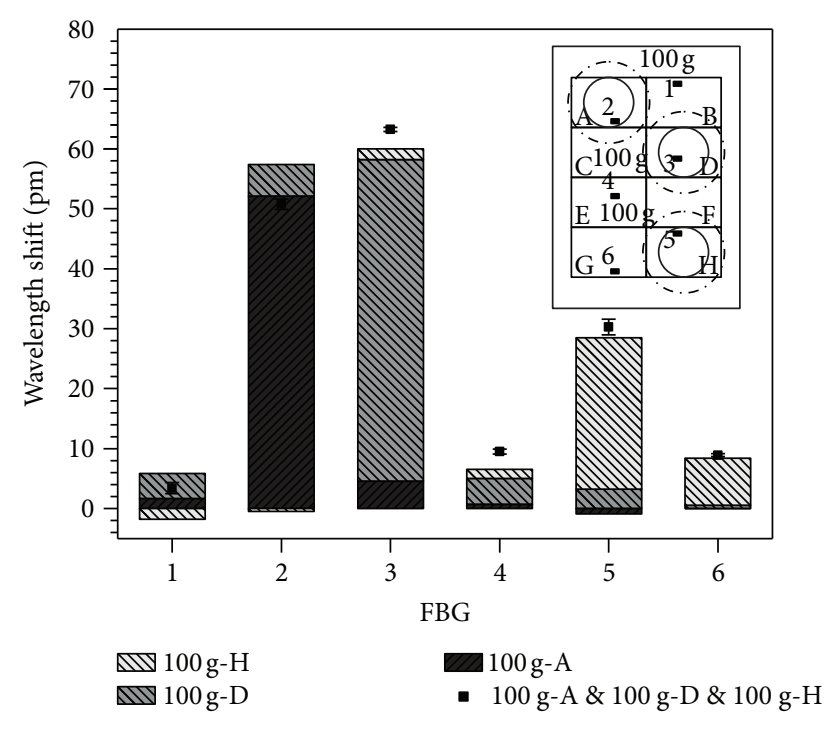

FIGURE 8: Sensor responses to loads applied individually (stack columns) and simultaneously (square symbols) on cells A, D, and $\mathrm{H}$. The inset indicates the position of the loads in a diagram of the TSA.

$18.514 \mathrm{~dB}$ was obtained for the test with an average error of $14.833 \%$ (Figure 9(a)). The best reconstruction shown in Figure 9(b) provided an average error of $4.500 \%$ and SNR of $28.318 \mathrm{~dB}$. For three loads, the total average error was $8.117 \%$ and the average SNR was $23.279 \mathrm{~dB}$.

For the tests with four loads, Figures 9(c) and 9(d) show the worst and best reconstruction cases, respectively. The worst reconstruction resulted in an average error of $17.000 \%$ and a SNR of $7.768 \mathrm{~dB}$. On the other hand, for the best reconstruction, an average error of $3.500 \%$ and a SNR of $28.097 \mathrm{~dB}$. For four loads, the total average error was $10.488 \%$ and the average SNR was 19.660 for a reconstruction ratio of $90 \%$. Two configurations resulted in SNR lower than $12 \mathrm{~dB}$. One of these cases is indicated by arrows in Figure 9(c). The reconstruction for a load applied at cell A provided a wrong load recovering at cell $\mathrm{C}$.

Table 3 shows the average quality of the reconstruction, the relative error, and the reconstruction ratio for all tested configurations.

The results in Table 3 indicate that the increase in the number of the applied loads compromises the sparsity required for the application of the SDE method impairing the reconstruction quality. The complete reconstruction process takes less than 1 second, allowing TSS application in real-time sensing.

Considering the sensing area of $60 \times 80 \mathrm{~mm}$ divided in 8 cells, the spatial resolution is $30 \times 20 \mathrm{~mm}$.

The TSS metrological characteristics shown in Table 4 are obtained by combining the TSA (see Table 2) and SDE parameters. The system resolution for the determination of the applied force is given by the capacity of reconstruction of the SDE method.

The circular-shaped contact elements produce small wavelength shifts in the Bragg wavelengths that are compensated with a reference spectrum. Figures and tables of this section show results obtained with experiments realized at
$22.0 \pm 0.5^{\circ} \mathrm{C}$. Nevertheless, the capability of reconstruction was also tested with the TSA and the loads in thermal equilibrium at $17.5 \pm 0.5^{\circ} \mathrm{C}$ and $27.0 \pm 0.5^{\circ} \mathrm{C}$ without impairing the system performance.

The ability of detecting four loads simultaneously applied to the TSA opens the possibility of mapping forces produced by the touch of the hand. Figure 10 shows the performance of the TSS when four fingers simultaneously touch the TSA.

As the body skin and the silicone have low thermal conductivity and the silicone shows slow thermal expansion, the TSA performance is not impaired by heat transfer when the contact with the fingers occurs during a short-time interval of a few seconds.

This application shows that the TSS of this work could be used for rehabilitation of the hand or any other real-time application that requires recognizing the touch of the fingers.

\section{Conclusions}

The FBG encapsulation with silicone allows manufacturing a flexible TSA that can be directly attached to surfaces with different shapes. Furthermore, the silicone encapsulation is economically attractive and the production takes 24 hours without the need for special cure processes.

The FBG-coupled responses, allied to the linearity of the system, allow the TSA application in quasi-distributed sensing with a reduced number of the sensors.

The TSA operation is based on the measurement of the induced wavelength shifts of the six FBGs with respect to the reference wavelength values measured just before the force application. Therefore, as the system recognizes a pattern in the wavelength shifts experienced by the set of FBGs, the reconstruction performance is not affected by the temperature of operation if all system is in thermal equilibrium with the environment. Localized changes of temperature can negatively affect the TSA performance; however, the influence of temperature changes in the sensor responses is reduced by the encapsulation due to the low value of thermal conductivity [21] and the slow thermal expansion of the silicone elastomer. In applications involving the identification of the fingers' touch, as the skin also has low thermal conductivity, the TSA performance is not impaired by localized heat transfers when the contact occurs during a short-time interval of a few seconds.

Reconstruction of up to four simultaneous loads in real time with an average SNR of $19.66 \mathrm{~dB}$ and relative error lower than $10.5 \%$ was demonstrated with the SDE method developed by Negri et al. [8]. For more demanding applications, it is possible to reduce this error by increasing the number of sensors or by adjusting the size and number of cells, as long as the sparsity requirements are satisfied.

The method of encapsulation allows the production of a flexible and robust array of sensors embedded in a unique block of silicone that represent a considerable improvement compared to the rigid steel plate used in [8].

The advantages of the silicone elastomer combined with the 3D printing technology and the outstanding characteristics of the FBGs result in tactile sensor array that can be used 

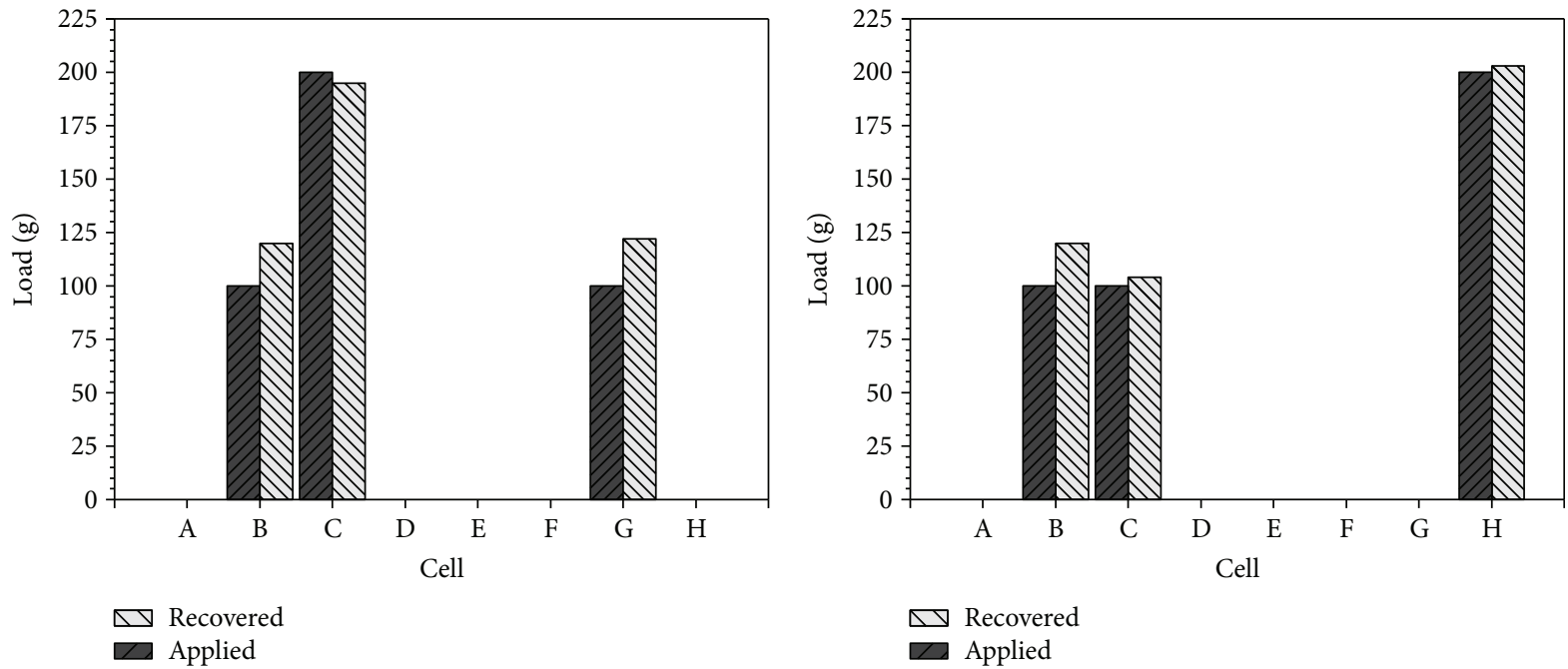

(a)

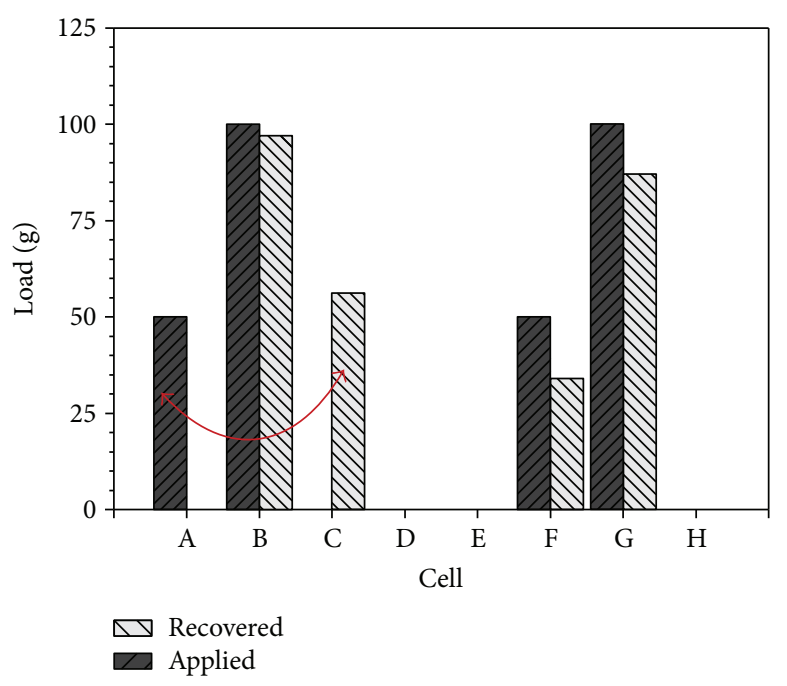

(c) (b)

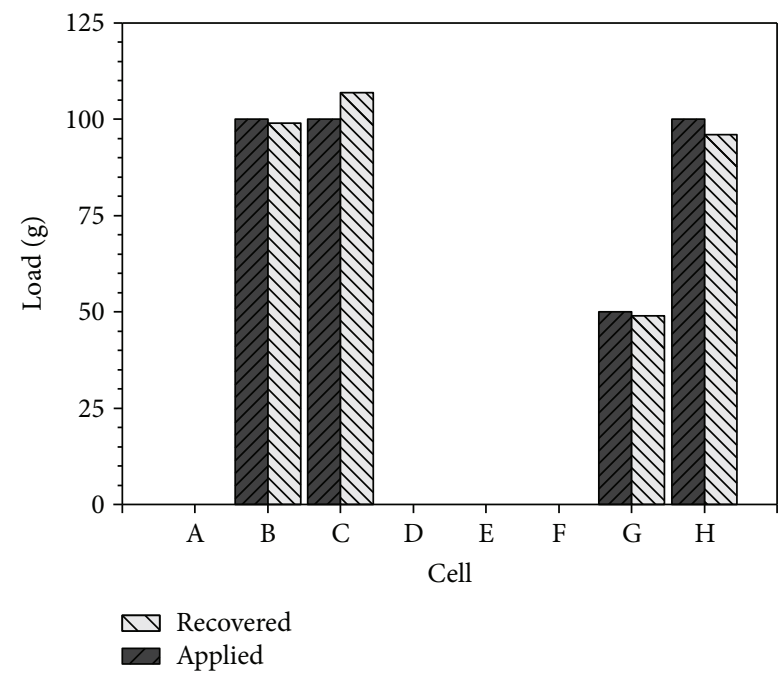

(d)

FIGURE 9: Reconstruction results for loads simultaneously applied to the TSA: (a) the worst and (b) best configurations for three loads; (c) the worst and (d) best configurations for four loads. One of the cases where the SNR was lower than $12 \mathrm{~dB}$ is indicated by arrows in (c).

TABLE 3: Average SNR, relative error, and reconstruction ratio for the reconstruction tests with application of up to four loads.

\begin{tabular}{lcccc}
\hline Number of loads & SNR $(\mathrm{dB})$ & SEM $(\mathrm{dB})$ & Error $(\%)$ & RR (\%) \\
\hline 1 & 25.440 & 0.998 & 8.775 & 100 \\
2 & 25.170 & 0.854 & 7.363 & 100 \\
3 & 23.279 & 0.573 & 8.117 & 100 \\
4 & $\mathbf{1 9 . 6 6 0}$ & $\mathbf{1 . 0 8 2}$ & $\mathbf{1 0 . 4 8 8}$ & $\mathbf{9 0}$ \\
\hline
\end{tabular}

TABLE 4: Metrological characteristics of the tactile sensing system.

\begin{tabular}{lc}
\hline Metrological characteristics & TSS \\
\hline Measuring interval $(\mathrm{g})$ & $25-250$ \\
Size $(\mathrm{mm})$ & $60 \times 80$ \\
Spatial resolution $(\mathrm{mm})$ & $30 \times 20$ \\
Error $(\%)$ & $<10.5$ \\
\hline
\end{tabular}

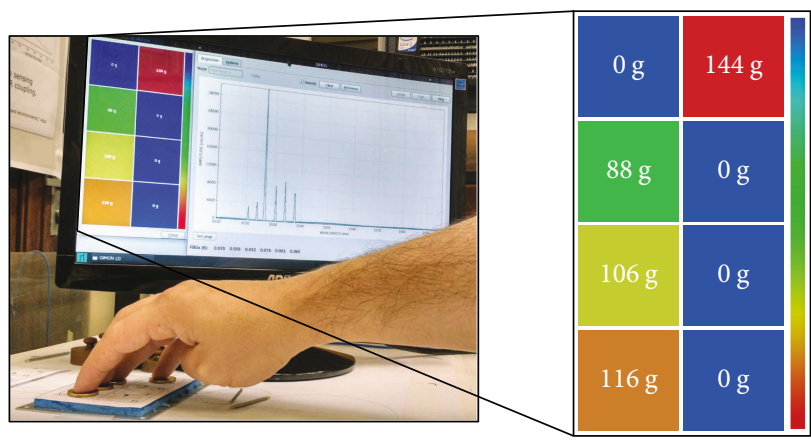

Figure 10: Picture showing a real-time reconstruction resulting from the touch of four fingers at the TSA. 
in a large number of applications, including biomedical and medical areas, mainly in the rehabilitation of the hand.

\section{Conflicts of Interest}

The authors declare that there are no conflicts of interest regarding the publication of this paper.

\section{Acknowledgments}

This work was supported by the Brazilian agencies CAPES, CNPq, FINEP, and Fundação Araucária.

\section{Supplementary Materials}

Examples of the tactile system operation are shown in two videos. One of them shows the spatial mapping of four loads and, in the other one, the TSA realizing real-time touch sensing can be seen. (Supplementary Materials)

\section{References}

[1] J.-S. Heo, J.-H. Chung, and J.-J. Lee, "Tactile sensor arrays using fiber Bragg grating sensors," Sensors and Actuators A: Physical, vol. 126, no. 2, pp. 312-327, 2006.

[2] G. T. Kanellos, G. Papaioannou, D. Tsiokos, G. Nianios, and N. Pleros, "Two dimensional polymer-embedded quasidistributed FBG pressure sensor for biomedical applications," Optics Express, vol. 18, no. 1, pp. 179-186, 2010.

[3] M. E. H. Eltaib and J. R. Hewit, "Tactile sensing technology for minimal access surgery-a review," Mechatronics, vol. 13, no. 10, pp. 1163-1177, 2003.

[4] P. Saccomandi, C. M. Oddo, L. Zollo et al., "Feedforward neural network for force coding of an MRI-compatible tactile sensor array based on fiber Bragg grating," Journal of Sensors, vol. 2015, Article ID 367194, 9 pages, 2015.

[5] L. H. Negri, Y. Wang, A. S. Paterno, M. Muller, and J. L. Fabris, "Distributive load position estimation by smart optical sensing," in Annals of $16^{\circ}$ SBMO - Simpósio Brasileiro de Microondas e Optoeletrônica e $11^{\circ}$ CBMag - Congresso Brasileiro de Eletromagnetismo (MOMAG2014), vol. 1, pp. 359-362, Curitiba, Brazil, 2014.

[6] B. M. Cowie, D. J. Webb, B. Tam, P. Slack, and P. N. Brett, "Fibre Bragg grating sensors for distributive tactile sensing," Measurement Science and Technology, vol. 18, no. 1, pp. 138146, 2007.

[7] L. H. Negri, E. M. Schiefer, A. S. Paterno, M. Muller, and J. L. Fabris, "An approach to improve the spatial resolution of a force mapping sensing system," Measurement Science and Technology, vol. 27, no. 2, article 25103, 2016.

[8] L. H. Negri, A. S. Paterno, M. Muller, and J. L. Fabris, "Sparse force mapping system based on compressive sensing," IEEE Transactions on Instrumentation and Measurement, vol. 66, no. 4, pp. 830-836, 2017.

[9] M. A. Pedroso, L. H. Negri, M. A. Kamizi, J. L. Fabris, and M. Muller, "Fabrication of a tactile sensor array with fiber Bragg gratings using a 3D printed mold," in 2017 SBMO/IEEE MTT-S International Microwave and Optoelectronics Conference (IMOC), pp. 1-4, Aguas de Lindoia, Brazil, 2017.

[10] M. A. Kamizi, G. R. C. Possetti, M. Muller, and J. L. Fabris, "Fiber Bragg grating sensors probed by artificial intelligence to detect and localize impacts on structures," Journal of Microwaves, Optoelectronics and Electromagnetic Applications, vol. 14, pp. SI25-SI34, 2015.

[11] E. J. Candès, J. K. Romberg, and T. Tao, "Stable signal recovery from incomplete and inaccurate measurements," Communications on Pure and Applied Mathematics, vol. 59, no. 8, pp. 1207-1223, 2006.

[12] R. Tibshirani, "Regression shrinkage and selection via the Lasso," Journal of the Royal Statistical Society, Series B, vol. 58, no. 1, pp. 267-288, 1996.

[13] C. H. Chuang, M. S. Wang, Y. C. Yu, C. L. Mu, K. F. Lu, and C. T. Lin, "Flexible tactile sensor for the grasping control of robot fingers," in 2013 International Conference on Advanced Robotics and Intelligent Systems, pp. 141-146, Tainan, Taiwan, 2013.

[14] P. Saccomandi, L. Zollo, A. L. Ciancio et al., "Tactile piezoresistive sensors for robotic application: design and metrological characterization," in 2017 IEEE International Instrumentation and Measurement Technology Conference (I2MTC), pp. 1-6, Turin, Italy, 2017.

[15] F. Castelli, "An integrated tactile-thermal robot sensor with capacitive tactile array," IEEE Transactions on Industry Applications, vol. 38, no. 1, pp. 85-90, 2002.

[16] A. Othonos and K. Kalli, Fiber Bragg Gratings: Fundamentals and Applications in Telecommunications and Sensing, Artech House, Boston, MA, USA, 1999.

[17] K. O. Hill and G. Meltz, "Fiber Bragg grating technology fundamentals and overview," Journal of Lightwave Technology, vol. 15, no. 8, pp. 1263-1276, 1997.

[18] Xiameter, "Silicone rubber," November 2016, https://www. xiameter.com.br/pt/ExploreSilicones/Silicone_Rubber/Pages/ silicone-rubber.aspx.

[19] M. A. Kamizi, M. A. Pedroso, J. L. Fabris, and M. Muller, "Fabrication and characterization of fiber Bragg grating based sensors for force measurements," in 2017 SBMO/IEEE MTT-S International Microwave and Optoelectronics Conference (IMOC), pp. 1-6, Aguas de Lindoia, Brazil, 2017.

[20] G. R. C. Possetti, R. C. Kamikawachi, M. Muller, and J. L. Fabris, "Metrological evaluation of optical fiber gratingbased sensors: an approach towards the standardization," Journal of Lightwave Technology, vol. 30, no. 8, pp. 10421052, 2012.

[21] J. Nedoma, M. Fajkus, L. Bednarek, J. Frnda, J. Zavadil, and V. Vasinek, "Encapsulation of FBG sensor into the PDMS and its effect on spectral and temperature characteristics," Advances in Electrical and Electronic Engineering, vol. 14, no. 4, pp. 460-466, 2016. 


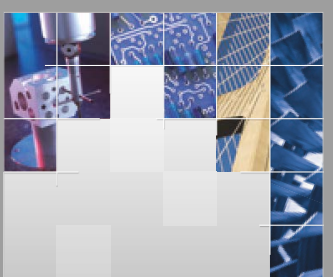

\section{Enfincering}
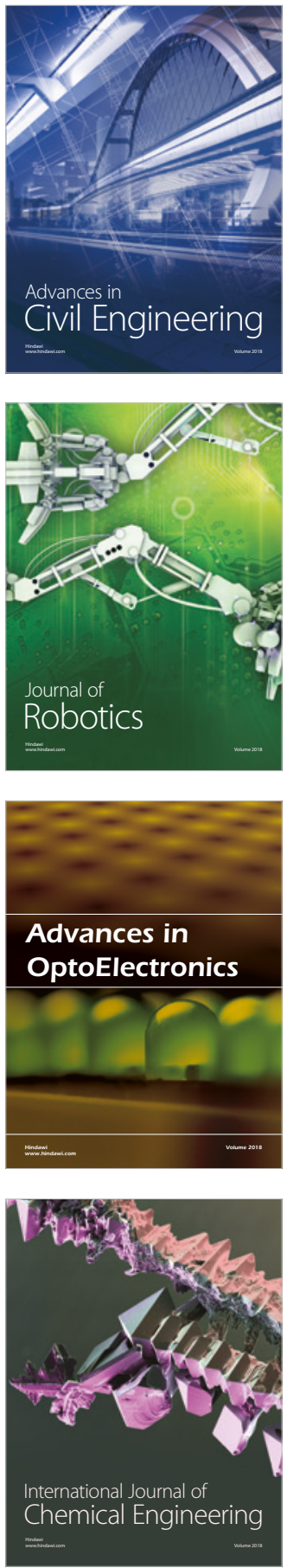

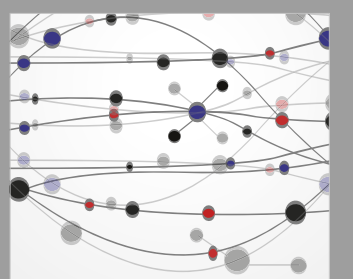

\section{Rotating \\ Machinery}

The Scientific World Journal

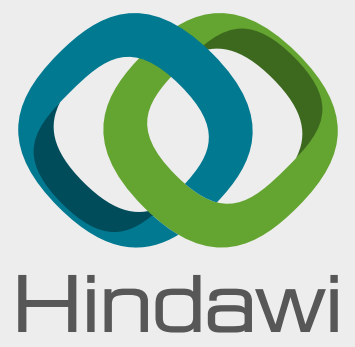

Submit your manuscripts at

www.hindawi.com
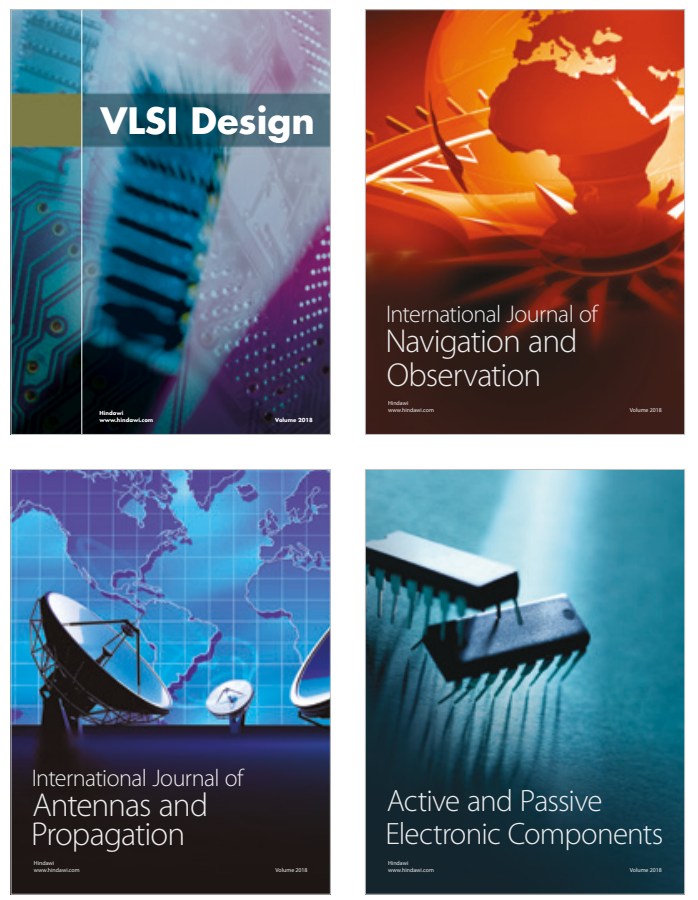
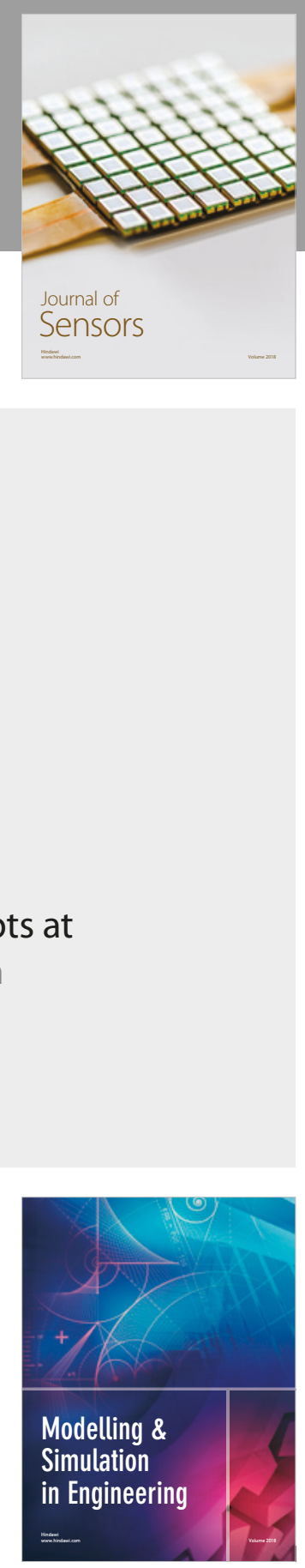

\section{Advances \\ Multimedia}
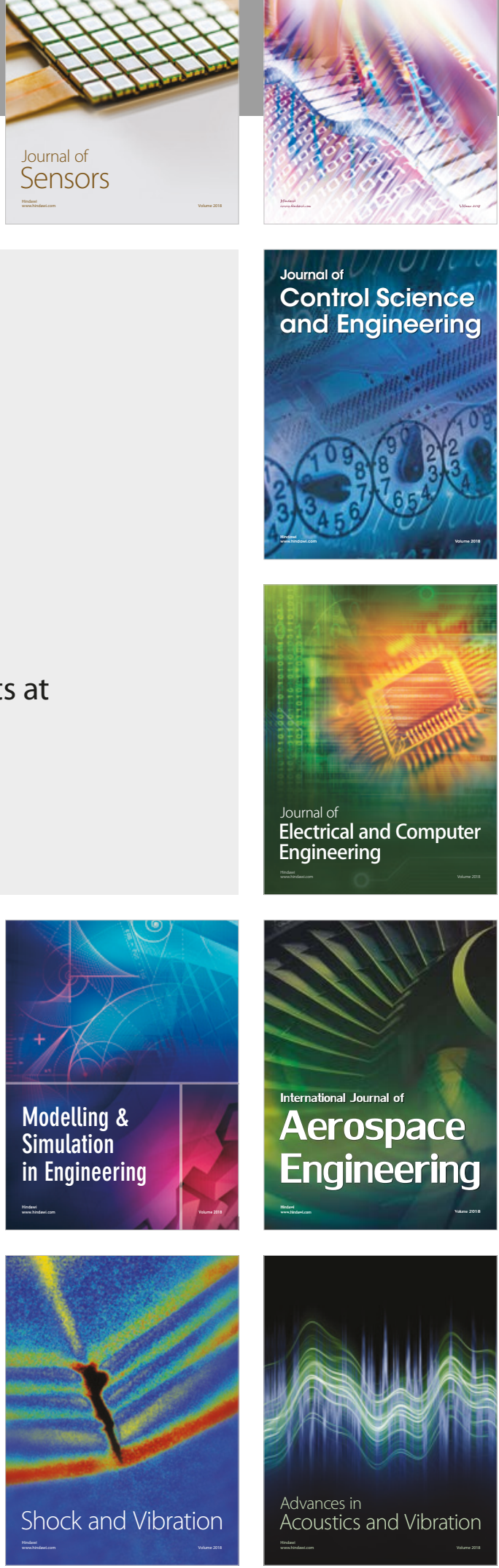